\title{
Applying the 2005 Canadian Hypertension Education Program recommendations: 1. Diagnosis of hypertension
}

ß See related article page 508

$\stackrel{+}{\sigma} \mathbf{T}$ Typertension is estimated to 1 be the third leading cause 苞 of death worldwide. Although 22\% of adult Canadians have hypertension, only $16 \%$ have it controlled with drug therapy ${ }^{1}$ $\stackrel{\circ}{\circ}$ even though numerous studies have proven the benefit of lowering blood pressure. A recent study showed that a delay of 3 months in effective blood pressure control in high-risk patients (those with evidence of target organ damage, diabetes mellitus, chronic kidney disease or macrovascular damage) is associated with at least a 2 -fold increase in cardiovascular morbidity and mortality. ${ }^{2}$ Therefore, the 2005 Canadian Hypertension Education Program recom-

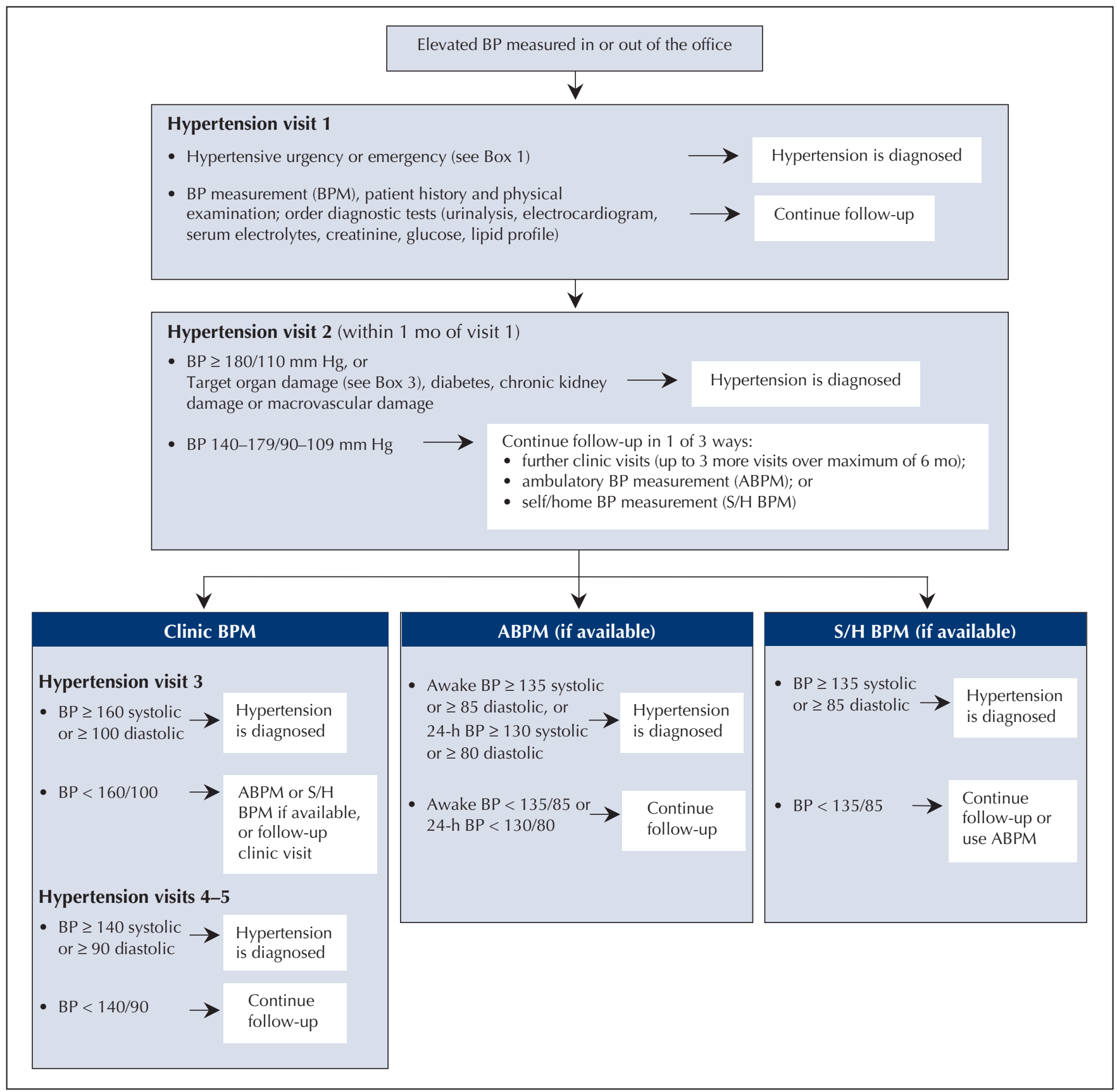

Fig. 1: The 2005 Canadian Hypertension Education Program recommendations for the assessment and diagnosis of hypertension. Patients with an elevated blood pressure measured during visits 4-5 may still have white-coat (office-induced) hypertension. 
mendations ${ }^{3}$ have been revised to expedite diagnosis in adults by (a) reducing the number of visits needed to establish the presence of hypertension in high-risk patients and in those with severe (stage 2) hypertension and (b) introducing the option of using self/home or ambulatory blood pressure monitoring in making the diagnosis.

Although diagnosis is related to a certain level of blood pressure, it also takes into account other factors, including target organ damage and concomitant cardiovascular risk factors. For adults (excluding those with diabetes or chronic kidney disease), an office blood pressure reading of $140 / 90 \mathrm{~mm} \mathrm{Hg}$ has a similar risk to a mean daytime (awake) ambulatory reading or a mean self/home blood pressure reading of 135/85 mm Hg.

The 3 hypothetical cases described in this article demonstrate how the new diagnostic al- gorithm can be applied to detect hypertension in adults (Fig. 1).

\section{Peter Bolli}

Hotel Dieu Grace Hospital

Windsor, Ont.

Martin Myers

Sunnybrook and Women's College

Health Sciences Centre

Toronto, Ont.

Donald McKay

Memorial University of Newfoundland

St. John's, Nfld.

For the Canadian Hypertension

Education Program
CASE 1: A previously healthy 52-year-old man sees his family physician because of a dog bite. His blood pressure is 196/102 $\mathrm{mm} \mathrm{Hg}$.

Question: Does this patient have hypertension?

Comment: The patient's blood pressure is clearly elevated, but an abnormal reading during a single visit does not establish the presence of hypertension unless there is a hypertensive urgency or emergency (Box 1). Pain may have contributed to the elevated blood pressure. Therefore, further readings at subsequent visits are necessary.

The patient is scheduled for another visit in 1 week's time (first hypertension-specific visit) (Fig. 1). At this visit, his blood pressure is $182 / 100 \mathrm{~mm} \mathrm{Hg}$. The remainder of the physical examination yields normal findings, and there are no exogenous risk factors that could have elevated the blood pressure (Box 2).
Question: Should this patient receive antihypertensive treatment now?

Comment: Not yet. However, because his blood pressure remains elevated, a further visit and investigations are required. ${ }^{3}$

At the patient's second hypertension-specific visit, 2 weeks later, his blood pressure is $182 / 112 \mathrm{~mm} \mathrm{Hg}$. Results of urinalysis and an electrocardiogram (ECG) are normal, as are his lipid profile and levels of serum electrolytes, creatinine and glucose.

Question: Can hypertension now be diagnosed and treatment started even though there is just an elevated blood pressure without target organ damage (Box 3 ) or other risk factors?
Box 2: Substances that can induce or aggravate hypertension

- NSAIDs

- Corticosteroids, anabolic steroids

- Oral contraceptives, sex hormones

- Vasoconstrictive, sympathomimetic decongestants

- Calcineurin inhibitors (cyclosporin, tacrolimus)

- Erythropoietin and analogues

- Monoamine oxidase inhibitors

- Licorice

- Cocaine, caffeine and other stimulants

- Alcohol (excessive intake)
Box 1: Hypertensive urgencies and emergencies

- Diastolic blood pressure $>130 \mathrm{~mm} \mathrm{Hg}$ or systolic blood pressure $>$ $200 \mathrm{~mm} \mathrm{Hg}$

- Accelerated or malignant hypertension with papilledema

- Hypertensive encephalopathy

- Intracerebral or subarachnoid hemorrhage

- Acute aortic dissection

- Acute refractory left ventricular failure

- Renal crisis from collagen vascular diseases

- Pheochromocytoma

- Rebound hypertension following cessation of clonidine

- Eclampsia

- Severe hypertension in patients requiring emergency surgery

- Severe epistaxis

\section{Box 3: Target organ damage}

Macrovascular

- Coronary artery disease

- Left ventricular hypertrophy

- Left ventricular systolic dysfunction

- Stroke, transient ischemic attack, cerebrovascular dementia

- Aortic and peripheral arterial disease

Microvascular

- Hypertensive nephropathy

- Hypertensive retinopathy 
Comment: Yes. Because this patient's systolic blood pressure remains above $180 \mathrm{~mm} \mathrm{Hg}$, hypertension can now be diagnosed (Fig. 1). ${ }^{3}$ The greatest fall in blood pressure occurs between the first and second visits. The power to distinguish between patients with and those without hypertension is $80 \%$ between visits 1 and 2 , and a further $10 \%$ between visits 2 and 3. Thereafter, fluctuations in blood pressure are mainly random. ${ }^{4}$ Therefore, it is unlikely that this patient's blood pressure would be normal on further visits. Persistent systolic blood pressure readings greater than $180 \mathrm{~mm} \mathrm{Hg}$ (or diastolic readings greater than $110 \mathrm{~mm} \mathrm{Hg}$ ) represent severe hypertension, and delaying treatment may increase the risk of a cardiovascular event. If this patient was at high risk (had evidence of target organ damage, diabetes, chronic kidney disease or macrovascular damage), the diagnosis would have applied even if his blood pressure was above 140/90 $\mathrm{mm} \mathrm{Hg}$ over 2 visits (Fig. 1). ${ }^{2}$
CASE 2: A 72-year-old woman visits her family physician for new prescriptions. Her blood pressure is $178 / 72 \mathrm{~mm} \mathrm{Hg}$. She mentions that she went to a walk-in clinic, where she had been given diclofenac $75 \mathrm{mg} 3$ times daily for back pain. The diclofenac therapy is stopped, and the patient is scheduled for a visit in 1 month.

Question: Does this patient have hypertension?

Comment: It is too early to decide. Although her blood pressure is elevated, hypertension cannot be diagnosed on the basis of a single reading during 1 visit, especially since the NSAID could have raised her blood pressure (Box 2).

At the next visit, which is considered the first hypertensionspecific visit, the patient's blood pressure is $168 / 72 \mathrm{~mm} \mathrm{Hg}$. Findings on the physical examination are otherwise normal. The patient is given a requisition for an ECG and laboratory tests and is asked to return in 1 month.

Question: Because the patient's blood pressure remains elevated, can hypertension be diagnosed and treatment started?

Comment: Any hypertensive effect of the diclofenac therapy should have worn off by now. Therefore, the patient may indeed have hypertension, but it is still too early to make this diagnosis with certainty. Hence, further investigation and follow-up are required.

At the patient's second hypertension-specific visit, her blood pressure is $160 / 68 \mathrm{~mm} \mathrm{Hg}$. The ECG shows sinus rhythm and a first-degree atrioventricular block. Laboratory results (serum electrolyte, creatinine and glucose levels, lipid profile, urinalysis) are all normal.

Question: Because the patient's blood pressure has remained elevated, can hypertension be diagnosed?

Comment: Not yet. Because her blood pressure is below 180/110 $\mathrm{mm} \mathrm{Hg}$ and there is no target organ damage, diabetes or chronic kidney disease, further follow-up of her blood pressure is required before a definitive diagnosis can be made. This can be done in 1 of 3 ways:

- further clinic visits (up to 3 more visits over a maximum of 6 months);

- ambulatory blood pressure monitoring; or

- self/home blood pressure monitoring. ${ }^{3}$

The patient opts for the third option. She is instructed in the use of an automated blood pressure measuring device and, after she demonstrates that she can use the device properly, is loaned one to take home. She is asked to take duplicate readings of her blood pressure twice a day for a week and to keep a log of the results. When she returns for her third hypertension-specific visit, the log shows blood pressure readings of $120 / 72$ to 130/78 $\mathrm{mm} \mathrm{Hg}$ (discarding the values of the first day). Her blood pressure at the clinic that day is $176 / 76 \mathrm{~mm} \mathrm{Hg}$.

Question: Can "white coat" (office-induced) hypertension be diagnosed?

Comment: Yes. The normal blood pressure readings (below $135 / 85 \mathrm{~mm} \mathrm{Hg}$ ) obtained at home establish this diagnosis. ${ }^{3}$ Patients do not always report readings accurately to their doctors. Devices that are capable of storing readings for the doctor to see are useful in eliminating this type of reporting bias.

However, the patient wants to be absolutely sure that her blood pressure is indeed normal and agrees to undergo ambulatory blood pressure monitoring. This method gives a daytime (awake) mean blood pressure reading of $128 / 70 \mathrm{~mm} \mathrm{Hg}$ and a 24-hour mean reading of 120/64 mm Hg, which confirms the diagnosis of office-induced hypertension. The patient is asked to return in 6 months' time for repeat ambulatory blood pressure monitoring and at yearly intervals thereafter, since office-induced hypertension may subsequently progress to hypertension. ${ }^{5}$ If the patient requires antihypertensive treatment any time later on, the presence of a first-degree atrioventricular block in the ECG would have to be considered when choosing the medication (e.g., caution should be used if selecting a $\beta$-blocker or nondihydropyridine calcium-channel blocker). 
CASE 3: A 53-year-old woman measures her blood pressure at a local drugstore because of headaches. Her blood pressure is $178 / 100 \mathrm{~mm} \mathrm{Hg}$. She returns to the drugstore 2 days later, and her blood pressure is 200/108 $\mathrm{mm} \mathrm{Hg}$. Worried about these readings, she arranges an urgent visit with her family physician, whom she sees the following day for her first hypertension-related visit (Fig. 1 ). At that visit, the patient's blood pressure is $168 / 96 \mathrm{~mm} \mathrm{Hg}$. Other findings on physical examination are normal. She is not taking hormone replacement therapy (Box 2).

Question: Can hypertension be diagnosed given the patient's high readings at the drugstore?

Comment: No, it would be premature to make this diagnosis. The readings obtained in the drugstore could be falsely high mainly because of a stress-induced hypertensive reaction or a nonstandardized condition under which the blood pressure was measured (e.g., the patient was in a hurry, or it was a cold day). Also, blood pressure measuring devices in some drugstores may not be reliable enough for clinical decisionmaking.
The patient is asked to return in 3 weeks, and she is given a requisition for laboratory tests and an ECG. At her second hypertension-specific visit her blood pressure is $176 / 100 \mathrm{~mm} \mathrm{Hg}$. The ECG is normal, as are the laboratory test results (serum electrolyte, creatinine and glucose levels, lipid profile and urinalysis). Question: Because the patient's blood pressure has remained elevated, can hypertension be diagnosed and treated?

Comment: Not yet. Hypertension can be diagnosed at this second visit only if her blood pressure is $180 / 110 \mathrm{~mm} \mathrm{Hg}$ or higher or if target organ damage, diabetes, chronic kidney disease or macrovascular damage is present. ${ }^{3}$ Therefore, the patient's elevated blood pressure needs to be confirmed by additional measurements, which can be taken at further clinic visits or by means of ambulatory or self/home blood pressure monitoring. ${ }^{3}$

The patient opts for ambulatory blood pressure measurement because she is anxious to know as soon as possible whether she has hypertension. It gives a daytime (awake) mean blood pressure of $170 / 98 \mathrm{~mm} \mathrm{Hg}$ and a 24-hour mean blood pressure of 166/96 mm Hg.

Question: Can hypertension be diagnosed?

Comment: Yes. A daytime (awake) mean blood pressure of $135 / 85 \mathrm{~mm} \mathrm{Hg}$ or greater, or a 24-hour mean blood pressure of 130/80 mm Hg or greater, obtained by ambulatory blood pressure measurement, establishes the diagnosis. ${ }^{3}$

\section{Competing interests: None declared.}

\section{References}

1. Joffres MR, Ghadirian P, Fodor JG, Petrasovits A, Chockalingam A, Hamet P. Awareness, treatment and control of hypertension in Canada. Am 7 Hypertens 1997;10:1097-102.

2. Julius S, Kjeldsen SE, Weber M, Brunner HR, Ekman S, Hansson L, et al. Outcomes in hypertensive patients at high cardiovascular risk treated with regimens based on valsartan or amlodipine: the VALUE randomized trial. Lancet 2004;363:2002-31.

3. Hemmelgarn BR, McAlister FA, Myers MG, McKay DW, Bolli P, Abbott C, et al. The 2005 Canadian Hypertension Education Program (CHEP) recommendations for the management of hypertension: Part 1 - Blood pressure measurement, diagnosis, and assessment of risk. Can 7 Cardiol 2005; 21(8):645-56.

4. Perry HM Jr., Miller JP. Difficulties in diagnosing hypertension: Implications and alternatives. 7 Hypertens 1992;10:887-96.

5. Palatini PP, Dorigatti F, Roman E, Gioinazzo P, Piccolo D, De Venuto G, et al. White-coat hypertension. A selection bias? F Hypertens 1998;16:977-84. 